\title{
Necrosis of the thumb after inadvertent injection of diclofenac in the radial artery: a case report
}

This article was published in the following Dove Press journal: Journal of Pain Research

\author{
Hyun-Chul Shon \\ Ji-Kang Park \\ Sang-Woo Kang \\ Jae-Young Yang
}

Department of Orthopaedic Surgery, College of Medicine, Chungbuk National University, Cheongju, Korea

Correspondence: ji-Kang Park

Department of Orthopaedic Surgery, 776

I Sunhawn-ro, Heungdeok-gu, Cheongju

28644, Korea

Tel +82432696077

Fax +82 432748719

Email carm0916@hanmail.net

\begin{abstract}
Inadvertent intra-arterial drug injection occurs rarely, but it can cause very serious clinical complications, and thus, awareness of inadvertent intra-arterial drug injection is needed. The complications mainly result from iatrogenic reasons and can occur because of vascular variations in the arteries, under circumstances where normal intravenous injection is difficult, or in drug abusers who perform self-injection. The adverse effect associated with intra-arterial drug injection is serious and may lead to necrosis, requiring amputation of the affected extremity, infection, pseudoaneurysm, rhabdomyolysis, compartment syndrome, and permanent disability. However, the etiology of such adverse effects has not been clearly identified and treatment methods have not yet been established. We encountered a patient who developed necrosis of the thumb after an inadvertent injection of diclofenac sodium in the radial artery due to variations in the forearm arteries. Here, we report the prevention and treatment of, and precautions against, the dangers of intra-arterial drug injection.
\end{abstract}

Keywords: intra-arterial drug injection, diclofenac sodium, thumb necrosis

\section{Introduction}

Since the report by Macintosh in 1943 about the complications arising from an inadvertent intra-arterial injection of thiopental, the dangers of intra-arterial drug injection have been reported in various papers. ${ }^{1}$ Intra-arterial drug injections mostly occur accidentally, mainly as iatrogenic complications during anesthesia procedures. ${ }^{2}$ The adverse effects, which were reported to occur in up to $29 \%$ of patients, are serious, including necrosis, leading to amputation of the affected extremity, infection, pseudoaneurysm, rhabdomyolysis, and compartment syndrome. ${ }^{2}$ However, the etiology of such adverse effects has not been clearly identified and treatment methods have not been established yet. Therefore, prevention of inadvertent intra-arterial drug injection is the most important solution to this problem. Persons who belong to the high-risk group for inadvertent intra-arterial drug injection include obese individuals whose veins are difficult to find, drug abusers who perform self-injection without any anatomical knowledge, and those with vascular variations in the forearms. ${ }^{3} \mathrm{We}$ had encountered a case of necrosis of the thumb after an intra-arterial injection of diclofenac sodium in an artery that was mistaken for a vein in a patient with arterial variation in the forearm. In this study, we report the dangers of intra-arterial drug injection, and the prevention and treatment of its associated effects, along with a literature review. 


\section{Case}

A 63-year-old male patient $(180 \mathrm{~cm}, 75 \mathrm{~kg}$, retired office worker, Korean) was admitted with the chief complaint of necrosis of the left thumb. He was healthy and a non-smoker with no underlying disease, including vascular disease, diabetes, or hypertension and family history. One week before admission, he visited a private clinic because of a cold, and received an injection of diclofenac sodium (OS-beta, Unimed $^{\circledR}$ ) $90 \mathrm{mg}$ in the left wrist. He developed numbness and severe pain in the distal part of the wrist immediately upon receiving the injection, as well as hypesthesia in the thumb and index finger after several hours. On the following day, he developed swelling and cyanosis of the thumb and index finger. Necrosis of the distal end of the thumb started developing from the third day after the injection. Subsequent ultrasonography at another hospital indicated reduced blood flow to all fingers of the left hand and no measurable blood flow in the left thumb. Consequently, the patient was prescribed beraprost $40 \mathrm{mg}$ twice a day. Even though he was taking the medication, his symptoms and necrosis worsened, and he visited our hospital after becoming aware of the possibility of needing an amputation. At the time of admission to our hospital, necrosis from the interphalangeal joint to the distal end on the left thumb was observed and an injection mark was visible in the proximal portion of the radial styloid process (Figure 1). But there was no abnormality in the patient's laboratory data. Strong pulse was palpable in the proximal portion of the radial styloid process of both wrists, but not where the radial artery is generally located. Variation in the superficial dorsal antebrachial artery was identified on angiographic CT (Figure 2). After confirming the boundary of the necrotic area, the proximal phalanx was amputated. Thumb reconstruction was not performed because the patient chose not to undergo the procedure.

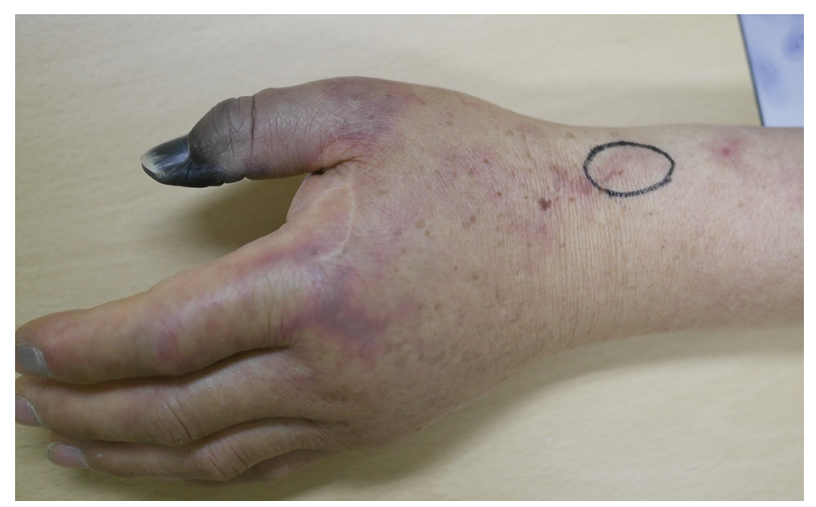

Figure I At the time of admission, necrosis of the left distal thumb phalanx was observed and an injection mark (black circle) was visible in the proximal portion of the radial styloid process.

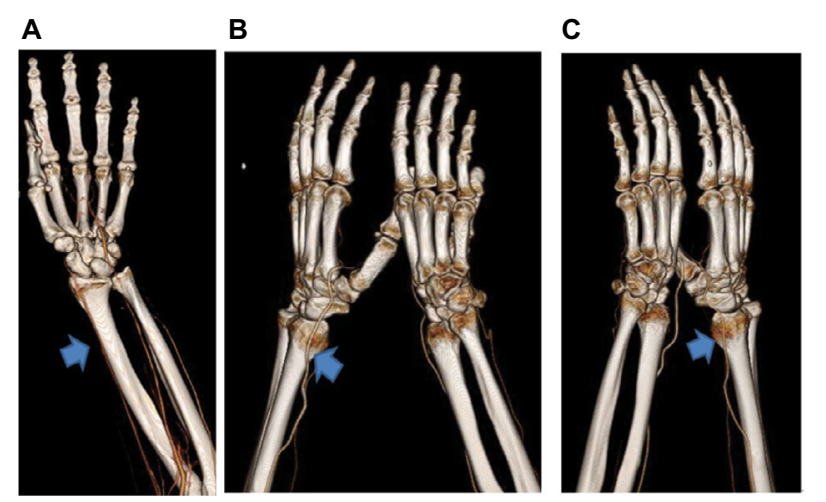

Figure 2 (A) The radial artery normally passes through the entire radial bone (arrow). (B) and (C) In the present patient, angiographic computed tomography revealed a variation of the radial artery where it was coursing above the styloid process as a superficial dorsal antebrachial artery (arrows).

Written informed consent was obtained from the patient for publication of this case report and any accompanying images.

\section{Discussion}

Since the introduction of thiopental in 1943, other reported drugs for intra-arterial injection have included psychotropic drugs or anesthetics such as promethazine, propofol, diazepam, methohexital, depomedrol, and benzodiazepine. ${ }^{1}$ Intraarterial drug injections mostly occur because of iatrogenic complications during anesthesia procedures, as well as in drug abusers who perform self-injection without any anatomical knowledge. ${ }^{2}$ However, normal arterial drug injection is used for the treatment of femoral artery pseudoaneurysm, chemotherapy for some tumors and monitoring catheter during anesthesia or in the intensive care unit. Diclofenac sodium, which was injected in the patient in this case, is a nonsteroidal anti-inflammatory drug commonly used in clinical settings, and usually administered through intramuscular or intravenous injection. The known adverse effects of intramuscular injection of diclofenac include rhabdomyolysis and myopathy. ${ }^{4}$ Samanta and Samanta ${ }^{5}$ and Kumar et $\mathrm{al}^{6}$ reported cases of amputation due to necrosis of the finger after an intra-arterial injection of diclofenac in a radial artery mistaken for a vein. The rate of amputation of an extremity due to the adverse effects of intra-arterial drug injection is $29 \%$. Among such cases, the rate of amputation resulting from intra-arterial benzodiazepine injection is high (up to $50 \%$ ). ${ }^{2}$

The risk factors of inadvertent intra-arterial drug injection include obesity, which makes the veins difficult to find; self-injection by drug abusers with no anatomical knowledge; and vascular variations in the forearm. Juric et al ${ }^{7}$ introduced the subcutaneous vein detection solution using near-infrared spectroscopy. This is the most recent technology to become 
available that can be used as a guidance tool to facilitate vein identification. It is especially beneficial for high-risk populations. Among the high-risk populations, variations in the forearm radial artery are the most common cause of inadvertent intra-arterial injection. The most common variation in the forearm radial artery is a high-rising radial artery that forms superficial branches, terminates in the thenar region or binds with the ulnar artery to form a palmar arch that eventually forms superficial palmar branches. ${ }^{7}$ Another variation of the radial artery involves the superficial dorsal antebrachial artery that forms an incomplete palmar arch between the thumb and index finger. ${ }^{8}$ This crosses the cephalic vein on the superficial portion of the radial styloid process, causing it to be mistaken for an intravenous injection site. Among radial artery variations, superficial dorsal antebrachial artery variation has been reported to have a prevalence of $\sim 1 \%$. As it shows no specific symptoms, it may not be detected unless angiography is performed. Brown et $\mathrm{al}^{9}$ performed angiography in patients with superficial dorsal antebrachial artery variation and reported that $\sim 13 \%$ of the patients had an incomplete form of such variation, in which the superficial palmar arch that supplies blood to the thumb does not anastomose with the ulnar artery. In such cases, when drug is injected in the radial artery or vascular damage occurs, necrosis of the thumb may occur because of the absence of blood supply to the ulnar artery. In our case, we believe that as the superficial dorsal antebrachial artery, which branches in the forearm, passed above the radial styloid process, it was mistaken for the cephalic vein, which is commonly used for intravenous injections.

Symptoms that appear after intra-arterial drug injection include acute pain, numbness, and burning sensation in the peripheral and distal areas from the injection site, as well as neurosensory symptoms such as an abnormal sensation. Pseudoaneurysm, rhabdomyolysis, and compartment syndrome and tissue necrosis may occur within a few days and cause permanent functional damage. ${ }^{3}$ Of the total patients, $78 \%$ had severe pain, $37 \%$ had neurological symptoms, and $17 \%$ had terminal necrosis. ${ }^{2}$ Although the exact mechanism of such tissue damage has not been identified, vasoconstriction, thrombus formation, crystallization, endothelial inflammatory response, cytotoxicity response, and hyperosmolarity have been reported as possible causes. ${ }^{3}$ Therefore, the treatment decision should be based on the mechanism, including the use of an arterial vasodilator, anticoagulant therapy, selective use of thrombolytic agent, use of inflammatory antagonist, and steroid therapy, whereas prophylactic antibiotics should be used to prevent infection. ${ }^{2,3}$ Devulapalli et $\mathrm{al}^{2}$ reported an amputation rate of $4 \%$ within $14 \mathrm{~h}$ of intra-arterial drug injection and an amputation rate of $46 \%$ after $14 \mathrm{~h}$.

Inadvertent intra-arterial drug injection can cause serious adverse effects such as necrosis and amputation of the ends of the extremities, regardless of the drug; however, the etiology of such adverse effects has not been identified and treatment methods have not been established yet. Therefore, the best option would be to identify the associated risk factors and symptoms, along with the prevention of inadvertent intraarterial drug injection and treatment of its adverse effects upon early detection. In the present case, severe pain and numbness were identified immediately after the injection. Had these signs been used to suspect intra-arterial drug injection and appropriate treatment administered early, the possibility of amputation could have been reduced.

\section{Conclusion}

Clinically, it is not easy to prevent inadvertent intra-arterial drug injection. Therefore, early detection of an event and initiation of treatment is a way to reduce the amputation rate of distal extremities. Although the subsequent treatments such as pain control, anticoagulation, rehabilitation, and specific therapy are relatively standardized methods, the correlation with pathophysiology remains unclear.

\section{Disclosure}

The authors report no conflicts of interest in this work.

\section{References}

1. Keene JR, Buckley KM, Small S, Geldzahler G. Accidental intra-arterial injection: a case report, new treatment modalities, and a review of the literature. J Oral Maxillofac Surg. 2006;64(6):965-968.

2. Devulapalli C, Han KD, Bello RJ, LaPorte DM, Hepper CT, Katz RD. Inadvertent intra-arterial drug injections in the upper extremity: systematic review. J Hand Surg Am. 2015;40(11):2262-2268.e5.

3. Sen S, Chini EN, Brown MJ. Complications after unintentional intraarterial injection of drugs: risks, outcomes, and management strategies. Mayo Clin Proc. 2005;80(6):783-795.

4. Selimoglu O, Basaran M, Ugurlucan M, Ogus TN. Rhabdomyolysis following accidental intra-arterial injection of local anesthetic. Angiology. 2009;60(1):120-121.

5. Samanta Sukhen, Samanta Sujay. Accidental intra arterial injection of diclofenac sodium and their consequences: report of two cases. Anaesth Pain Intensive Care. 2013;17(1):101-102.

6. Kumar M, Singh J, Sharma P, Khera A, Singh P. Accidental intraarterial injection of diclofenac -case report. J Clin Diagn Res. 2015;9(1):PD16-PD17.

7. Juric S, Flis V, Debevc M, Holzinger A, Zalik B. Towards a low-cost mobile subcutaneous vein detection solution using near-infrared spectroscopy. Sci World J. 2014;2014:365902.

8. Gonzalez-Compta X. Origin of the radial artery from the axillary artery and associated hand vascular anomalies. J Hand Surg Am. 1991;16(2):293-296.

9. Brown MJ, Edstrom LE, Zienowicz RJ. A symptomatic radial artery anomaly and its surgical treatment. J Hand Surg Am. 1999;24(1):178-181. 
The Journal of Pain Research is an international, peer reviewed, open access, online journal that welcomes laboratory and clinical findings in the fields of pain research and the prevention and management of pain. Original research, reviews, symposium reports, hypothesis formation and commentaries are all considered for publication.
The manuscript management system is completely online and includes a very quick and fair peer-review system, which is all easy to use. Visit http://www.dovepress.com/testimonials.php to read real quotes from published authors. 